\title{
Beta Decays and the Inner Structures of the Neutrino in a NLHV Design
}

\author{
Dirk J. Pons ${ }^{1}$, Arion D. Pons ${ }^{1} \&$ Aiden J. Pons ${ }^{2}$ \\ ${ }^{1}$ Department of Mechanical Engineering, University of Canterbury, Christchurch, New Zealand \\ ${ }^{2}$ Rangiora New Life School, Rangiora, New Zealand \\ Correspondence: Dirk J. Pons, Department of Mechanical Engineering, University of Canterbury, Private Bag \\ 4800, Christchurch 8020, New Zealand. E-mail: dirk.pons@canterbury.ac.nz
}

Received: March 24, 2014 Accepted: April 8, 2014 Online Published: April 17, 2014

doi:10.5539/apr.v6n3p50 URL: http://dx.doi.org/10.5539/apr.v6n3p50

\begin{abstract}
A novel conceptual theory is developed for the beta decay and electron capture processes, based on the specific non-local hidden-variable (NLHV) design provided by the Cordus theory. A new mechanics is sketched out for the interactions of particules through their discrete forces, and is a deeper level representation of Feynman diagrams. The new mechanics is able to correctly predict the outcomes of the decay processes, beta minus, beta plus, electron capture. It predicts specific NLHV structures for the neutrino and antineutrino. The velocity and unique spins of the neutrino species may then be explained as a consequence of the hidden structures.
\end{abstract}

Keywords: weak interaction, ontological, HED mechanics

\section{Introduction}

The beta decay processes are important in nucleosynthesis at genesis, stellar physics, and the behaviour of nuclides. The empirical aspects of decay may be well quantified, but there are significant ontological gaps in the explanations. Why does the neutron need to decay in the first place? Given that it is stable in the nucleus of the atom, why does it decay outside? What does the neutron comprise that it is able to split into three independent particles (e.g. in $\beta$ - decay to a proton, electron, and antineutrino)? These questions cannot be answered by Quantum mechanics or the Standard particle model of physics. Nonetheless addressing these foundational questions has the potential to lead to a better understanding of the interaction between the proton and neutron, and ultimately to a theory that better explains the nuclides and their decays.

This paper addresses this topic, with the particular area under examination being the neutron decays and the production processes for neutrinos. The work is a conceptual extension of a particular non-local hidden-variable (NLHV) design called the Cordus conjecture (D. J. Pons, A. D. Pons, A. M. Pons, \& A. J. Pons, 2012). We show that consideration of neutron decay provides a candidate design for the internal structure of the neutrino. In turn this explains several otherwise enigmatic aspects of neutrino behaviour.

\section{Decay Processes, Neutrinos, and the Weak Interaction}

\subsection{Neutron Decay}

The neutron was identified by Chadwick (1932) only recently in history. The beta minus decay itself had been known earlier (Rutherford, 1911), including that the emission product was an electron (Thomson, 1904), but was not immediately attributed to neutron decay. The other product of the decay, the neutrino, was postulated by Pauli (1930) and Fermi (Fermi, 1934; Wilson, 1968). The decay process has been empirically quantified, and the attributes of the particles are likewise known (quantum numbers), but an ontological explanation is mostly lacking.

Key particles that emerge specifically in the context of weak decays are the neutrino species and the W bosons, and these have their own ambiguities. The Standard model of physics proposes that the weak force is one of four fundamental interactions, the others being electromagnetism, gravitation, and the strong force. The weak force is said to be transmitted by $\mathrm{W}$ and $\mathrm{Z}$ bosons, and the effects of the force are diverse. The $\mathrm{W}$ bosons transmute quarks between the $\mathrm{u}$ and $\mathrm{d}$ families (hence change the flavour of a quark), and thereby also convert between protons and neutrons (hence change in charge). The $\mathrm{Z}$ boson is the mechanism for momentum transfer between 
particles. The $\mathrm{W}$ bosons are experimentally observed, are heavy, short-ranged, and short-lived. However the weak $\mathrm{W}$ interaction is more of an agent for transmutation rather than a force, as it does not bind particles together or cause them to move relative to each other as all the other interactions do. The empirical approaches, based on high-energy impacts, produce accurate quantitative models of the decay channels, but are quite unable to explain the decay processes (ontologically deficient). In addition these models are local to the situation, and do not give insights into other situations (poor external validity).

Neutrino species are commonly produced in decay processes. The mechanisms for their creation are unknown. These particles are problematic because they interact little with matter, so detection is more difficult than other particles, and consequently their attributes are incompletely understood. The low reactivity of the neutrino with other matter implies that it does not respond to the strong force. However it does to the weak: indeed it practically defines the weak interaction. In the standard model it was initially believed that neutrinos would be massless, because they are all left-spin-handed. No right-spin-handed neutrinos have been detected. This absence plus the requirement for conservation of angular momentum at formation, requires the left-handed neutrino to travel at the speed of light, and for the neutrino to be massless. Thus they should not respond to gravitation, i.e. not interact with the hypothesised Higgs boson. The neutrino may change generation while in transit, which is termed oscillation. The conventional explanation is that the three generations, which have different masses, are in coherent superposition within any one neutrino. This requires the neutrinos to have a small mass, which is incongruent with other reasoning for massless neutrinos. This is something of a challenge for the standard model (Murayama, 2002). How the mass might arise is uncertain. Since neutrinos are always left-handed, there does not seem to be an easy way for the Higgs boson to provide mass, unless right-handed neutrinos (and left-handed antineutrinos) are added to the Standard Model. However, these sterile neutrino particles have not been observed. Another conjecture is that the neutrino is its own antiparticle and thereby obtains mass through the Majorana effect. However the magnitude of this is doubtful. So the question of neutrino mass, and the mechanisms thereof, is still an open question.

Consequently there is still a great deal that is unknown about the neutrinos. These questions are not merely curiosities, since the neutrino is implicated as being involved in many of the unsolved areas of fundamental physics and cosmology. If the behaviour of neutrinos and antineutrinos is different (asymmetrical), then it could help explain why charge-parity $(\mathrm{CP})$ violation occurs, and explain why there is more matter than antimatter in the universe. The properties of neutrinos, particularly mass and handedness, might point to new physics. They may be involved in the dark matter problem. They are also important in theories of cosmology, for example some string theories propose superluminal sterile neutrinos. At a more practical level, they are probes for the interior of stellar objects, since they are not appreciably blocked by the outer layers of stars, nor interstellar dust, and therefore may help develop better theories for stellar evolution.

This whole system, comprising multiple decay processes, $\mathrm{W}$ bosons, and neutrino-species, is well quantified, but lacks ontological explanations. There is a need for theories that better explain the neutrino phenomena, the decay processes that produce them, and the cosmological behaviour of neutrinos.

\section{Purpose and Approach}

The purpose of this work was to see whether the non-local hidden-variable (NLHV) sector permits the creation of a new physical theory for the physics of nuclear decay. This is not a promising starting point as there has been no coherent development in this sector since the de-Broglie-Bohm theory (de Broglie, 1925; Bohm \& Bub, 1966), which is generally considered defunct. Also, there are a number of reasons, briefly summarised below, why there is generally low confidence in the hidden sector.

Bell's theorem implies that either superluminal effects or local hidden-variables do not exist (Bell, 1964). The empirical evidence favours entanglement behaviours, hence non-locality, and thus local hidden-variable solutions are considered precluded. Even so there is some doubt as not all loopholes for "mutual rapport" between particles (Bell, 1964) have been excluded. Much empirical work has gone into closing those loopholes with increasingly tighter tests. It is generally accepted that these tests are stringent enough to exclude just about all feasible local hidden-variable solutions. All the same, Bell's theorem does not exclude hidden-variable theories of the non-local kind (NLHV). Additional theoretical work has subsequently been performed in an attempt to explore the feasibility of NLHV solutions. For example, a certain sub-type of NLHV theories ("crypto-nonlocal", with particles that are independent to each other) has been excluded (Leggett, 2003; Romero et al., 2010). Other sub-types have also been excluded on theoretical grounds (Groblacher et al., 2007; Gisin, 2011). However these analyses are not without their critics, who have pointed out potential unreliability in the cases against NLHV solutions (Laudisa, 2008). It is therefore realistic to state that while local hidden-variable 
solutions are almost certain not to exist, non-local solutions are still not excluded (De Zela, 2008). Part of the difficulty is finding more candidate NLHV solutions to evaluate, as the obvious designs have been identified, evaluated, and generally been found wanting. There is a certain stasis in the field, since the theoreticians cannot conclusively prove the non-viability of the entire NLHV sector, and the conceptual thinkers have run out of specific candidates to offer for evaluation. Consequently it is believed that if a NLHV solution exists, it is likely to be counterintuitive (Groblacher et al., 2007). Importantly, the inequalities do not preclude NLHV solutions entirely. This is not contentious.

We approached this task using the systems-engineering design methodology, which is ideally suited to conceptual development ab initio. The systems engineering part of the methodology provides the framework that ensures that there is a logical consistency between sub-theories and the whole, as the development proceeds. The design part of the methodology is used to infer the requisite internal structures of a system, from its external functionality. In the present situation the external functionality is represented by the empirically observed behaviour of the system. In this context the 'system' is the nuclear decays and their inputs and outputs. This is a novel way of thinking about fundamental physics, and has already been successful in creating a new physics around a NLHV design (Pons et al., 2012; D. J. Pons, A. D. Pons, \& A. J. Pons, 2013a, 2013b, 2013c, 2014b). The theory includes solutions for wave-particle duality, unification of the electro-magneto-gravitational and strong forces, explanations for antimatter and the process of annihilation, a solution that explains the table of nuclides ( $\mathrm{H}$ to $\mathrm{Ne}$ ), and a theory for time.

We started from the NLHV design provided by the Cordus particule structure (Pons et al., 2012). We then took the known beta nuclear decays, and applied the design method to infer a set of internal structures for the neutron and neutrino. The resulting solution is expressed as the discrete force arrangements of the particules. We deduced the mechanics for transmutation of particles, under this NLHV scenario. We devised a method to represent this mechanics and the state of each particule. This is called the HED mechanics, for reasons to be given. It represents particule interactions in this NLHV theory, like Feynman diagrams do for 0D points in QM. The HED mechanics has proved to be a useful construct, since it is versatile in application to other phenomena such as annihilation (Pons et al., 2014a) and the nuclides (Pons et al., 2013b). The annihilation work also provided an ontological explanation of the underlying mechanisms for mass-energy equivalence. This is especially relevant to the present paper, in that both annihilation and decay involve common principles: internal rearrangements of NLHV structures. The resulting theory naturally yields explanations for the velocity, non-reactivity, and selective spin attributes of the neutrino species.

\section{Results}

The present work is a logical extension of a prior concept for a NLHV design, and this is briefly explained first. Then we explain the proposed mechanics for manipulating discrete force structures, followed by application to the nuclear decays.

\subsection{Cordus Theory}

The Cordus theory has been described elsewhere, and is only briefly summarised here. The core conjecture is that all particles have inner and outer structures comprising two reactive ends some distance apart (span), connected by a fibril (hence cordus), and emitting discrete forces (Pons et al., 2012). This is called a particule to differentiate it from the zero-dimensional (0D) point idea of quantum mechanics (QM). The fibril is a persistent structure that provides instantaneous connectivity and synchronicity between the two reactive ends, but does not interact with matter. The reactive ends are energised sequentially (at the de Broglie frequency), during which they emit discrete forces out into the external environment. The locus of these over time defines a type of flux line called a hyperfine fibril (hence hyff). The discrete forces are emitted in three spatial directions (hence hyff emission directions, HEDs), and hence space is filled with a fabric of discrete forces (D. J. Pons \& A. D. Pons, 2013). The quantity, direction, and arrangement of these discrete forces determine the type of particule and are responsible for charge, mass, matter-antimatter species differentiation, and spin (Pons et al., 2014b). The discrete forces are responsible for the electro-magneto-gravitational and strong interactions, though the theory uses the term synchronous interaction in place of the strong, as this better describes the proposed nature of the interaction (Pons et al., 2013c).

\subsection{HED Mechanics}

The HED mechanics is a set of rules for the manipulation of discrete forces in this NLHV particule. These rules arise naturally from the core principle that a particule is defined by the pattern of discrete forces it emits, and therefore changes to the discrete forces cause the particule to change its nature. The pattern of discrete forces is represented in HED notation, which simply indicates the number of discrete forces in each of three orthogonal 
spatial directions $[r, a, t]$, their charge (negative: $x^{1}$, positive: $x_{1}$ ) and matter-antimatter hand (antimatter uses underscore, e.g. $\mathrm{x}_{1}$ ). The HED mechanics is the hidden-variable equivalent of Feynman diagrams for 0D points.

There are a number of assumptions in the HED mechanics, which are noted as lemmas. None are unreasonable, since they correspond to conservation principles that are already accepted in other physical theories. The first is that the HED mechanics requires the discrete forces to be conserved, rearranged, or even transformed, during transmutation and decay processes. Thus all discrete forces have to be accounted for, though they can be changed into other types as the annihilation theory shows (Pons et al., 2014a).

The second principle is that the HED mechanics allow a charge- and hand-neutral complex of discrete forces to be added to any particule. This neutral complex comprises $\mathrm{x}_{1.1}^{1.1}$ where $\mathrm{x}$ is one of the HED axes. The complex is represented symbolically by $\uparrow \downarrow$ where $\uparrow=\mathrm{x}_{1}{ }^{1}$ and $\downarrow=\mathrm{x}_{1}{ }^{1}$. Being charge- and hand-neutral, this complex has no net energy. It is analogous to QM's idea of a vacuum fluctuation. Note that neither a single discrete force (say) $\mathrm{x}^{1}$ nor a single pair (say) $\uparrow$ may be added to a particule ex vacuua: all such additions must be neutral as regards both charge and hand. Thirdly, the structure $\uparrow \uparrow \uparrow=\left[\mathrm{r}_{1}{ }^{1} \cdot \mathrm{a}_{1}{ }^{1} \cdot \mathrm{t}_{1}{ }^{1}\right]$ corresponds to a pair of photons, alternatively an electron-antielectron pair (Pons et al., 2014a). This set of discrete forces may be added to a particule as part of energy absorption.

The application of HED mechanics to a particule, or assembly of particules, is best understood as a remanufacturing process. The discrete forces are permitted to change to other axes (HEDs), and separate/combine into other groupings, and thereby redefine the identity of the particule. Next the HED mechanics are applied to the remanufacture of the neutron in its decay process.

\subsection{Quark, Neutron and Proton Structures}

\subsubsection{Quark Structure and Fractional Charge}

We propose the cause of fractional charge of quarks as selective activation of the three orthogonal HEDs, i.e. a directional charge (c.f. colour charge in quantum chromodynamics, QCD). We express those quarks in HED notation as:

$$
\begin{aligned}
& \text { u Quark u }\left(\mathrm{r}_{1} \quad \mathrm{a}_{1} \quad . t\right) \\
& \text { d Quark d }\left(\mathrm{r}^{1} \text {.a } . t\right)
\end{aligned}
$$

The allocation of the discrete forces to specific directions $[r, a, t]$ is nominal at this stage. The Cordus theory permits discrete forces to change emission directions (likewise QCD permits colour-change). The Cordus theory also explains why there are exactly three colour charges: because this is the number of geometric directions for emission of discrete forces. It also explains why colour is only seen in fractional charge situations: because there are none of the three emission directions may be unfilled in unit-charge particules.

\subsubsection{Proton and Neutron Overt Structures}

The overt HED structure of the neutron and proton are then determined from their quark content. The neutron structure is known to comprise one u quark and two d. Hence:

$$
\mathrm{n}(\mathrm{udd})=\mathrm{n}\left(\mathrm{r} \cdot \mathrm{a}_{1}{ }^{1} \cdot \mathrm{t}_{1}{ }^{1}\right)
$$

Likewise the proton structure is predicted to be:

$$
\mathrm{p}(\text { uud })=\mathrm{p}\left(\mathrm{r}_{1.1}^{1} \cdot \mathrm{a}_{1} \cdot \mathrm{t}_{1}\right)
$$

The above are the overt HED structures of the quarks and nucleons, as derived from considerations of fractional charge. Whether or not nucleons really comprises quarks is of little concern at this level, and it is sufficient to accept that the quark structures seen at high-energy impacts represent a dynamic regrouping of discrete force structures. The Cordus theory for the synchronous interaction permits particles to outwardly represent only their incomplete discrete force forces (hence overt structures), and conceal any balanced structures internally (covert structures). So the proton, neutron, and quarks may have additional discrete forces, not shown here.

The overt structures represent the net charge of the particule, and also engage in the synchronous (strong) interactions with other external particules. They are proposed to be important in nuclear bonding, as has been shown by extending the theory to the table of nuclides. In contrast the covert structures engage primarily with each other, internal to the particule. They are not involved in bonding, but do contribute to the mass. They may also be available for remanufacture into other particules.

\subsubsection{Covert Structures}

Considerations of the generations permits the covert structures of the quarks to be estimated. The generations can 
be conceptually explained by a design wherein the actual quark structures are as follow:

$$
\begin{aligned}
& \mathrm{u}\left[\left(\mathrm{r}_{1} \cdot \mathrm{a}_{1} \cdot \mathrm{t}\right)+\left[\downarrow_{1}{ }^{1}\right] \cdot\left[\uparrow^{1}{ }_{1}\right]\right.
\end{aligned}
$$

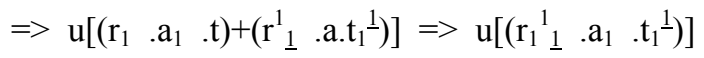

$$
\begin{aligned}
& \mathrm{d}\left[\left(\mathrm{r}^{1} \text {.a.t) }\right]+\left[\downarrow_{1}{ }^{1}\right] \cdot\left[\uparrow^{1}{ }_{1}\right]\right. \\
& \Rightarrow \mathrm{d}\left[\left(\mathrm{r}^{1} \cdot \mathrm{a} . \mathrm{t}\right)+\left(\mathrm{r}_{1}{ }^{1} \cdot \mathrm{a}^{1}{ }_{1}^{1} . \mathrm{t}\right)\right]=\mathrm{d}\left[\left(\mathrm{r}^{1}{ }_{1}{ }^{1} \cdot \mathrm{a}^{1}{ }_{1} \cdot \mathrm{t}\right)\right]
\end{aligned}
$$

It will be noted that the covert part is balanced in both charge (e.g. ${ }_{1}^{1}$ ), and matter-antimatter hand (e.g. ${ }^{1}{ }_{1}$ ), and it to this characteristic that its inertness is attributed.

This means that the full proton and neutron structures, which comprise $\mathrm{u}$ and $\mathrm{d}$ quarks are proposed as follow. For the neutron:

$$
\begin{aligned}
& \left.\mathrm{n}(\mathrm{udd})=\mathrm{n}\left[\mathrm{u}\left(\mathrm{r}_{1}^{1}{ }_{\underline{1}}^{1} \cdot \mathrm{a}_{1} \cdot \mathrm{t}_{1}\right)^{\frac{1}{}}\right)+\mathrm{d}\left(\mathrm{r}^{1}{ }_{1}{ }^{1} \cdot \mathrm{a}_{\underline{1}}^{1} \cdot \mathrm{t}\right)+\mathrm{d}\left(\mathrm{r}^{1} 1^{1} \cdot \mathrm{a}_{\underline{1}}^{1} \cdot \mathrm{t}\right)\right]
\end{aligned}
$$

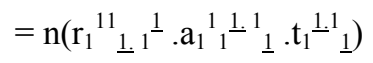

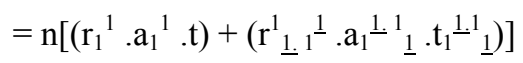

$$
\begin{aligned}
& =\mathrm{n}\left[\left(\mathrm{r}_{1}{ }^{1} \cdot \mathrm{a}_{1}{ }^{1} \cdot \mathrm{t}\right)+(\mathrm{r} \uparrow \downarrow \cdot \mathrm{a} \uparrow \downarrow \cdot \mathrm{t} \uparrow \downarrow)\right] \\
& =\mathrm{n}\left(\mathrm{r}_{1}{ }^{1} \cdot \mathrm{a}_{1}{ }^{1} \cdot \mathrm{t}\right)^{*}
\end{aligned}
$$

The first component $n\left(r_{1}{ }^{1} \cdot a_{1}{ }^{1} . t\right)$ is the overt part and interacts with the proton in the nucleus. This component is sufficient to explain nuclear structure (Pons et al., 2013b). The second part $\left(\mathrm{r}_{1.1}^{1} 1^{\frac{1}{2}} \cdot \mathrm{a}_{1} \mathbf{1}^{\frac{1}{1}}{ }_{1} \cdot \mathrm{t}_{1}{ }^{1.1}{ }_{1}\right)$ is balanced in HED, charge, and hand, and is therefore self-stable under the HED mechanics. This is abbreviated (*). This is the covert part, in that it does not interact explicitly in the synchronous interaction (strong force). However the emission of all these discrete forces contributes to an increase in mass, i.e. it contributes to the gravitational interaction (which is de-synchronous or discoherent). Later, when considering the decay of the free neutron, we raise the possibility that the free neutron shifts its discrete forces around to avert decay. Thus the bound and free states of the neutron are predicted to be slightly different, see Figure 1. This difference is not material to the present argument.

Likewise the proton structure is predicted to be:

$$
\begin{aligned}
& \mathrm{p}(\mathrm{uud})=\mathrm{p}\left[\mathrm{u}\left(\mathrm{r}_{1}{ }_{1}^{1} \cdot \mathrm{a}_{1} \cdot \mathrm{t}_{1}{ }^{\left.\frac{1}{}\right)}\right) \cdot \mathrm{u}\left(\mathrm{r}_{1}{ }_{1}^{1} \cdot \mathrm{a}_{1} \cdot \mathrm{t}_{1}{ }^{\frac{1}{}}\right) \cdot \mathrm{d}\left(\mathrm{r}^{1}{ }^{1}{ }^{\frac{1}{1}} \cdot \mathrm{a}^{1}{ }_{1} \cdot \mathrm{t}\right)\right] \\
& =\mathrm{p}\left[\left(\mathrm{r}_{1.1} \cdot{ }_{1}^{1.1}{ }_{1}^{1} \cdot 1^{1} \cdot \mathrm{a}_{1}{ }^{1}{ }_{1}^{1} \cdot 1^{1} \cdot \mathrm{t}_{1}{ }^{1} \cdot 1^{\frac{1}{1}}\right)\right.
\end{aligned}
$$

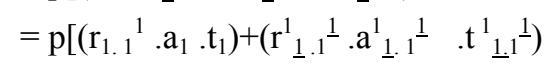

$$
\begin{aligned}
& =\mathrm{p}\left[\left(\mathrm{r}_{1.1}{ }^{1} \cdot \mathrm{a}_{1} \cdot \mathrm{t}_{1}\right)+(\mathrm{r} \uparrow \downarrow \cdot \mathrm{a} \uparrow \downarrow \cdot \mathrm{t} \uparrow \downarrow)\right] \\
& =\mathrm{p}\left(\mathrm{r}_{1.1}{ }^{1} \cdot \mathrm{a}_{1} \cdot \mathrm{t}_{1}\right)^{*}
\end{aligned}
$$




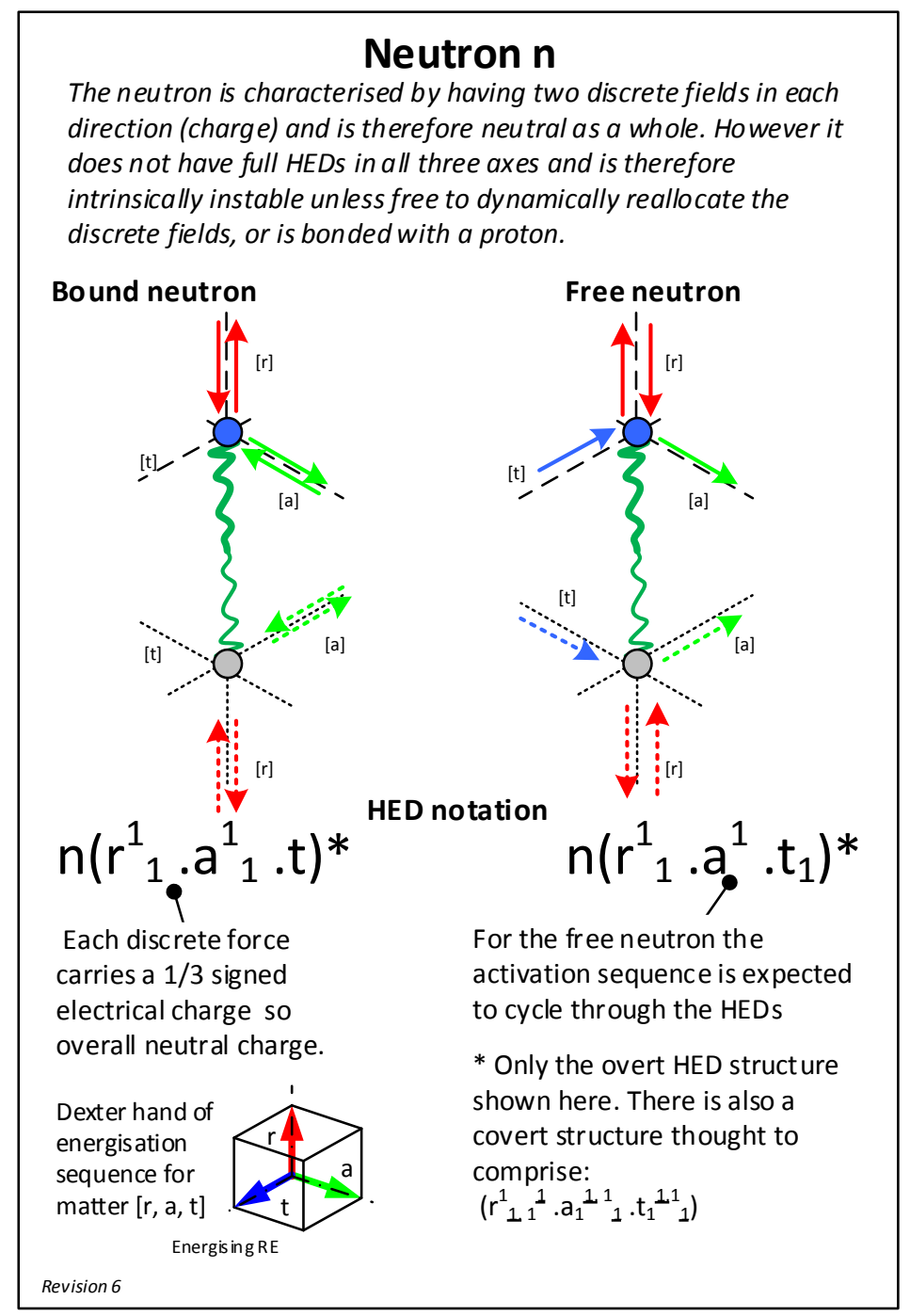

Figure 1. Proposed internal and external (discrete force) structures of the neutron

So the covert component $\left(^{*}\right)$ is the same for both the proton and neutron, is internally symmetrical. The proton structure is shown in Figure 2.

Next, we take these HED structures for the quarks and nucleons, and substitute into the beta decay processes, thereby inferring the HED structures of the neutrino and antineutrino.

\subsection{Beta-Decay and the Antineutrino (v) Structure}

In $\beta$ - decay, or electron emission, the free-neutron decays, after a relatively long life, into a proton, electron, and an electron antineutrino:

$$
\mathrm{n}=>\mathrm{p}+\mathrm{e}+\underline{\mathrm{v}}_{\mathrm{e}}
$$

$\beta$ - decay occurs spontaneously in nuclei that have too many neutrons relative to protons. We now represent this with HED notation, substitute the NLHV structures for the proton and electron, and solve for the antineutrino structure. We start with the neutron HED structure and rearrange. We only use the overt component, i.e. Equation 3 rather than Equation 7. This is because both the neutron and proton have the same covert component, so those structures are conserved and need not be considered. However to find enough discrete forces to manufacture the electron, the structure of which has previously been determined (Pons et al., 2014a), it is necessary to add another two neutral complexes, each being $\uparrow \downarrow=\mathrm{x}_{1}{ }^{1}+\mathrm{x}_{1}{ }^{1}$, to create a transitional assembly $\mathrm{O}$. Then rearrange to create groups of discrete forces corresponding to the known HED structures of the proton and electron. Distribute all the remaining discrete forces into a residual particule and identify this as the antineutrino: 


$$
\begin{aligned}
& \mathrm{n}\left(\mathrm{r}_{1}{ }^{1} \cdot \mathrm{a}_{1}{ }^{1} \cdot \mathrm{t}\right)+\left[\downarrow_{1}{ }^{\underline{1}}\right]\left[\uparrow^{1}{ }_{1}\right]+\left[\downarrow_{1}{ }^{\frac{1}{}}\right] \cdot\left[\uparrow^{1}{ }_{1}\right] \\
& \Rightarrow \mathrm{O}\left[\left(\mathrm{r}_{1}{ }^{1} \cdot \mathrm{a}_{1}{ }^{1} \cdot \mathrm{t}\right)+\left(\mathrm{r}_{1}^{1} \cdot \mathrm{a}_{1}{ }^{1}{ }^{1} \cdot \mathrm{t}^{1}\right)\right] \\
& =>\mathrm{O}\left[\left(\mathrm{r}_{1}{ }_{1}^{1}{ }^{\frac{1}{1}} \cdot \mathrm{a}_{1}{ }^{1} \cdot \mathrm{t}_{1}{ }^{1}\right)+\left(\mathrm{r}^{1} \cdot{ }_{1}^{1} \cdot \mathrm{a} \mathrm{t}^{1}\right)\right] \\
& =>O\left[\left(r_{1}^{1} \cdot a_{1} \cdot t_{1}\right)+\left(r^{1}{ }^{1} \cdot a^{1} \cdot t^{1} \underline{1}^{1}\right)\right] \\
& =>\mathrm{O}\left[\left(\mathrm{r}_{1}{ }_{1}^{1} \cdot \mathrm{a}_{1} \cdot \mathrm{t}_{1}\right)+\left(\mathrm{r}^{1} \cdot \mathrm{a}^{1} \cdot \mathrm{t}^{1}\right)+\left(\mathrm{r}_{\underline{1}}{ }^{1} \cdot \mathrm{a} \cdot \mathrm{t}_{\underline{1}}{ }^{\frac{1}{1}}\right)\right] \\
& =>\mathrm{p}\left(\mathrm{r}_{1}{ }_{1}^{1} \cdot \mathrm{a}_{1} \cdot \mathrm{t}_{1}\right)+\mathrm{e}\left(\mathrm{r}^{1} \cdot \mathrm{a}^{1} \cdot \mathrm{t}^{1}\right)+\underline{\mathrm{v}}\left(\mathrm{r}_{\underline{1}} \cdot{ }^{1} \cdot \mathrm{a} \cdot \mathrm{t}_{\underline{1}}{ }^{1}\right) \\
& \Rightarrow p+e+\underline{v}
\end{aligned}
$$

Thus the antineutrino is identified as having the HED structure:

antineutrino $\underline{\mathrm{v}}\left(\mathrm{r}_{\underline{1}} \underline{\underline{1}} \cdot\right.$ a. $\left.\cdot \underline{t}_{\underline{1}}\right)$

This identifies the antineutrino as being an antimatter particule, which is consistent with empirical evidence. Also, the analysis provides a particule with basic stability because all discrete forces have the same hand, and neutral charge, and this too is consistent with the known stability of the antineutrino. The particule has two negative charged discrete forces, and two positive. However the analysis cannot predict the allocation of discrete forces to specific HEDs with certainty. If we assume a different starting layout of the neutron e.g. $n\left(r_{1}{ }^{1} \cdot a_{1}{ }^{1} \cdot t\right)$, and proton e.g. $p\left(r_{1.1} \cdot a_{1}{ }^{1} \cdot t_{1}\right)$, then the predicted layout of the antineutrino also changes. However this does not undermine the principle. Furthermore the theory permits the allocation to be dynamic.

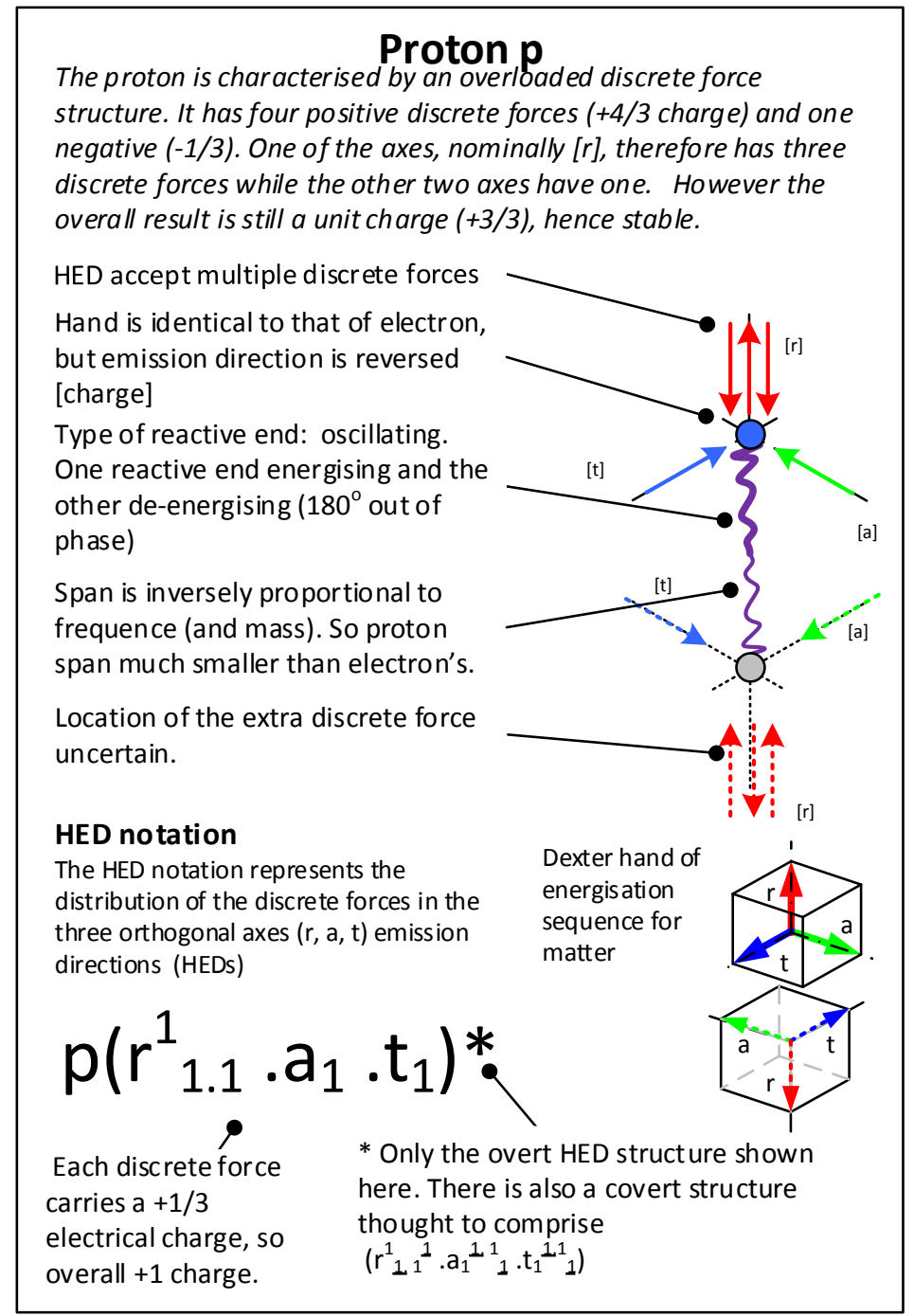

Figure 2. Proposed internal and external (discrete force) structures of the proton 


\subsubsection{Internal Design of the Antineutrino}

To further estimate the detailed layout of the antineutrino's discrete forces, we make use of the known fact that the antineutrino is mobile like the photon. According to the Cordus theory (Pons et al., 2014a) such mobility arises from lack of complete coverage of all three emission directions, and the resulting necessity to move relative to the discrete forces of the fabric (D. J. Pons \& A. D. Pons, 2013). The particule is propelled through the fabric by its imbalanced interaction with the fabric. Alternatively it may be considered to move through space if there are no pre-existing discrete forces (no fabric). These considerations allow us to estimate what the antineutrino might look like under this NLHV scenario, see Figure 3. This is one of several possible designs.

By the matter-antimatter Cordus theory (Pons et al., 2014a), we can already state the expectation that the neutrino is the corresponding mirrored HED structure. However this may be independently determined from consideration of the beta+ decay, as shown next. Both approaches give the same result, demonstrating that the approach has internal consistency.

\section{Antineutrino $\underline{\mathbf{v}}$}

The antineutrino has the same discrete force count as the neutrino, but the opposite hand of energisation sequence. The unique spin directions of the neutrino and antineutrino arise due to the hand differences.

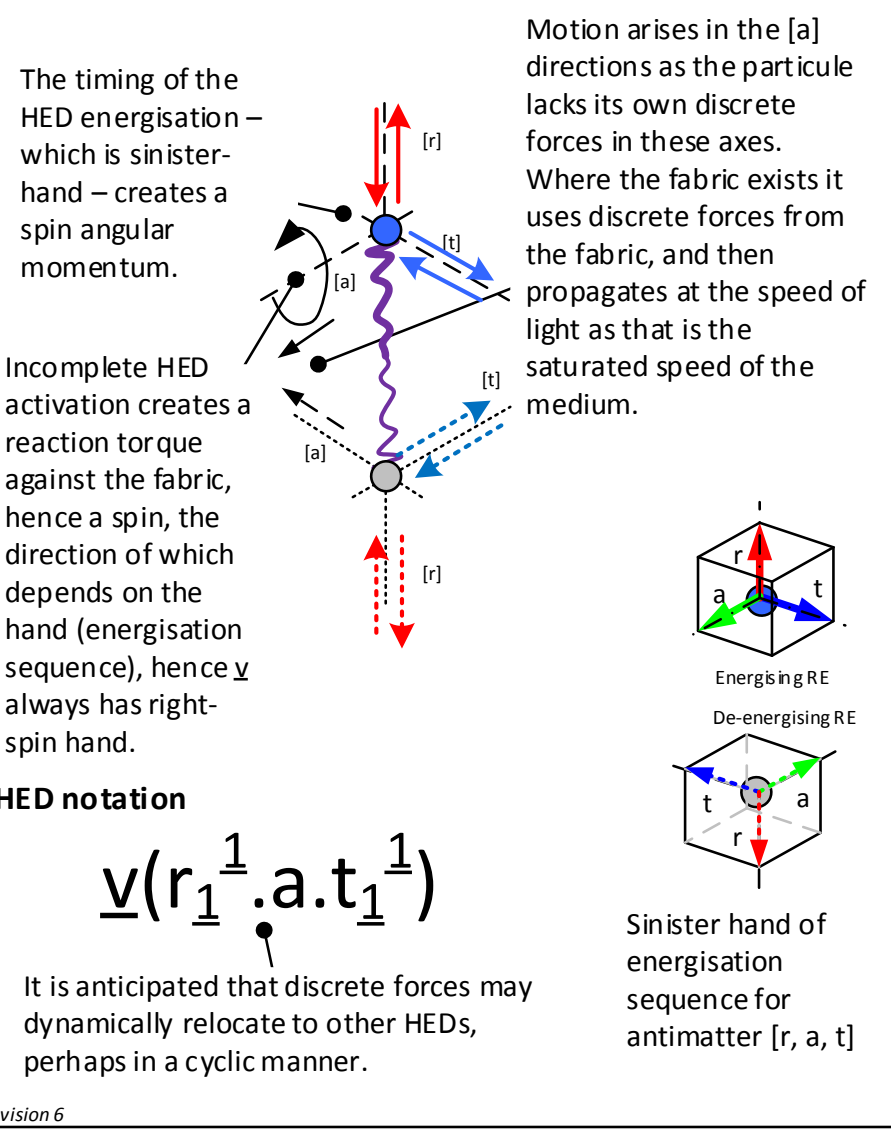

Figure 3. Predicted structure of the antineutrino

\subsection{Beta+ Decay and the Neutrino (v) Structure}

\subsubsection{Derivation of Neutrino Structure}

$\beta+$ decay, also called positron emission, occurs in proton rich nuclei and involves the conversion of an energetic proton into a neutron, antielectron (positron) and neutrino: 


$$
\mathrm{p}+\text { energy }=>\mathrm{n}+\underline{\mathrm{e}}+\mathrm{v}_{\mathrm{e}}
$$

We represent this in HED notation to derive the structure of the neutrino. We start by noting that adding energy to a particule can, under this framework, be considered the same as adding two photons (Pons et al., 2014a). This also adds more discrete forces into the system. These discrete forces are absorbed by the proton, to create an overloaded structure O. The external system, via the synchronous interaction (Pons et al., 2013c) at nuclear level (Pons et al., 2013b), drives the outcome towards a neutron. Hence those discrete forces necessary to define a neutron are retained, and the rest are placed into residual structures. The synchronous interaction, and the necessity for conservation of charge, forces this assembly into electron structures, hence an electron also emerges. The remaining discrete forces are placed into a waste structure. This does not permit further reduction, and is identified as the neutrino. Thus the process is:

$$
\begin{aligned}
& \mathrm{p}\left(\mathrm{r}_{1.1}{ }^{1} \cdot \mathrm{a}_{1} \cdot \mathrm{t}_{1}\right)+2 \mathrm{y}(\mathrm{r} \text { !.a.t }) \\
& =>\mathrm{O}\left[\left(\mathrm{r}_{1.1}{ }^{1} \cdot \mathrm{a}_{1} \cdot \mathrm{t}_{1}\right)+\left[\uparrow^{1}{ }_{1}\right]\left[\uparrow^{1}{ }_{1}\right]\left[\uparrow^{1}{ }_{1}\right]\right] \\
& =>\mathrm{O}\left[\left(\mathrm{r}_{1.1}{ }^{1} \cdot \mathrm{a}_{1}{ }_{1}^{1} \cdot \mathrm{t}_{1}\right)+\left(\mathrm{r}_{\underline{1}}^{1} \cdot \mathrm{a} \cdot \mathrm{t}_{\underline{1}}{ }^{1}\right)\right] \\
& =>\mathrm{O}\left[\left(\mathrm{r}_{1}{ }^{1} \cdot \mathrm{a}_{1}{ }^{1} \cdot \mathrm{t}\right)+\left(\mathrm{r}_{\underline{1} \cdot 1}^{1} \cdot \mathrm{a}_{\underline{1}} \cdot \mathrm{t}_{\underline{1.1}}^{1}\right)\right] \\
& =>\mathrm{O}\left[\left(\mathrm{r}_{1}{ }^{1} \cdot \mathrm{a}_{1}{ }^{1} \cdot \mathrm{t}\right)+\left(\mathrm{r}_{\underline{1}} \cdot \mathrm{a}_{\underline{1}} \cdot \mathrm{t}_{\underline{1}}\right)+\left(\mathrm{r}^{1}{ }_{1} \cdot \mathrm{a} \cdot \mathrm{t}^{1}{ }_{1}\right)\right] \\
& =>\mathrm{n}\left(\mathrm{r}_{1}{ }^{1} \cdot \mathrm{a}_{1}{ }^{1} \cdot \mathrm{t}\right)+\mathrm{e}\left(\mathrm{r}_{\underline{1}} \cdot \mathrm{a}_{\underline{1}} \cdot \mathrm{t}_{\underline{1}}\right)+\mathrm{v}\left(\mathrm{r}^{1}{ }_{1} \cdot \mathrm{a} \cdot \mathrm{t}^{1}{ }_{1}\right)
\end{aligned}
$$

Hence the neutrino structure is:

Neutrino $\mathrm{v}\left(\mathrm{r}^{1}{ }_{1} \cdot \mathrm{a} \cdot \mathrm{t}^{1}{ }_{1}\right)$

Note that this is equivalent to the overt neutron structure. However the difference between the neutron and neutrino is that the neutron also has a large covert component (Equation 7), whereas the neutrino has none. Likewise for the antineutrino. It is the covert component of the neutron that makes it heavier and static. The neutrino lacks a covert component, and its overt structure is incomplete, so it is required to be mobile.

The inferred neutrino structure is shown in Figure 4. This is consistent with the outcome from inverting the $\beta$ analysis. An interesting feature of this analysis is that it explains why the $\beta+$ process requires extra energy at the outset. This is because the energy corresponds to two photons hence $\uparrow \uparrow \uparrow$ (Pons et al., 2014a). In the conventional framework extra energy is typically interpreted as being applied to overcome some activation barrier. The present theory instead proposes that the energy goes into the creation of discrete forces. These participate in the remanufacture of the particule, and are therefore necessary at the outset. The two perspectives are complementary: the Cordus theory offers a mechanism whereby mass-energy equivalence contributes to particule interactions, which is not something that the energy perspective on its own can explain.

This design conceptually explains several known attributes of the antineutrino:

- moves at the speed of light

- exclusive spin

The primary focus of this analysis has been on the neutron decays and finding the identity of the neutrinos. The same HED mechanics may also be applied to the other decays, and for completeness these are provided below.

\subsection{Electron Capture}

In electron capture a proton absorbs an electron and converts to a neutron, emitting a neutrino.

$$
\mathrm{p}+\mathrm{e}=\mathrm{n}+\mathrm{v}
$$

This occurs in nuclei that have more protons than required for a stable state. Representing this in HED notation:

$$
\begin{aligned}
\mathrm{p}\left(\mathrm{r}_{1.1}{ }^{1} \cdot \mathrm{a}_{1} \cdot \mathrm{t}_{1}\right)+\mathrm{e}\left(\mathrm{r}^{1} \cdot \mathrm{a}^{1} \cdot \mathrm{t}^{1}\right) \\
\quad=>\mathrm{O}\left[\left(\mathrm{r}_{1.1}{ }^{1 \cdot 1} \cdot \mathrm{a}_{1}{ }^{1} \cdot \mathrm{t}_{1}{ }^{1}\right)\right] \\
\quad=>\mathrm{O}\left[\left(\mathrm{r}_{1}{ }^{1} \cdot \mathrm{a}_{1}{ }^{1} \cdot \mathrm{t}\right)+\left(\mathrm{r}_{1}{ }^{1} \cdot \mathrm{a} \mathrm{t}_{1}{ }^{1}\right)\right] \\
=>\mathrm{n}\left(\mathrm{r}_{1}{ }^{1} \cdot \mathrm{a}_{1}{ }^{1} \cdot \mathrm{t}\right)+\mathrm{v}\left(\mathrm{r}_{1}{ }^{1} \cdot \mathrm{a} \cdot \mathrm{t}_{1}{ }^{1}\right)
\end{aligned}
$$

The method correctly identifies that it is the neutrino rather than antineutrino that emerges. Note that only the overt structure of the neutron is shown here. 


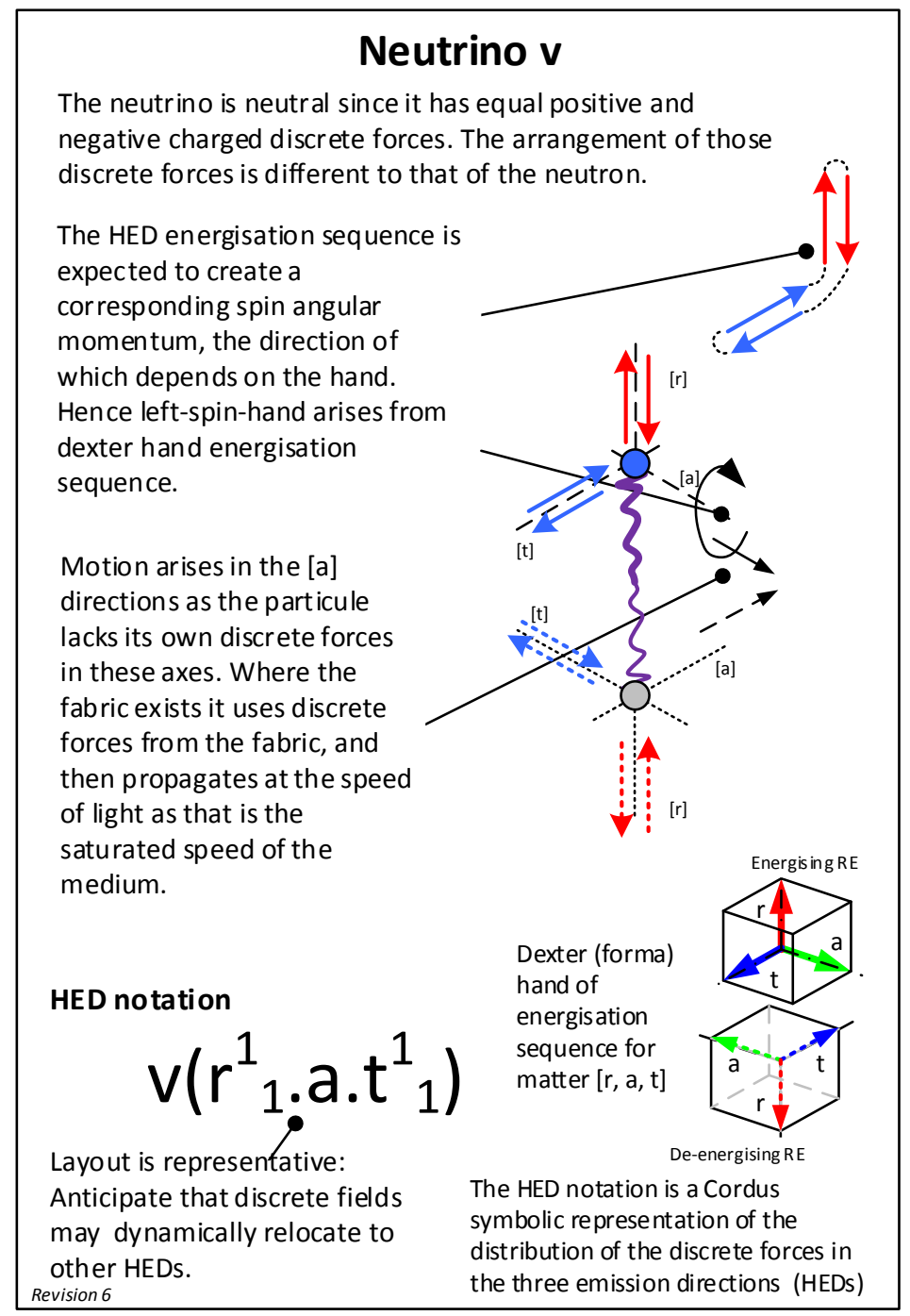

Figure 4. Predicted structure of the neutrino

Electron capture may involve one of the atom's own inner electrons, in which case there may be a cascade of consequences as the other electrons adjust, and this may result in a photon being emitted or an electron (Auger electron). Electron capture is known to occur when there is insufficient energy for decay via positron emission.

Compare the two proton decays:

$$
\begin{aligned}
& \beta+\text { decay: } p+\text { energy }=>n+\underline{e}+v_{e} \\
& \text { Electron capture: } p+e=>n+v
\end{aligned}
$$

We have already explained why $\beta+$ decay requires more energy - it needs a net increase in field structures to form the antielectron. Both processes allow the remanufacture of a proton into a neutron. The deeper question is: Why does the decay not always prefer the electron capture route, given that it does not require energy input?

The answer lies with the table of nuclides (Pons et al., 2013b), and the need for the unstable nuclear polymer to convert a proton into a neutron. This is a serious constraint for the nucleus, as the synchronous interaction (strong force) drives this outcome. Then $\beta+$ decay is a way of achieving the conversion, and also getting rid of unwanted binding energy. In comparison electron capture does not divest energy, but it does consume charge, and this is desirable in the fringes of stability. This is consistent with another part of the Cordus theory which explains photon emission as a process whereby a particule that cannot cope with its energy gets rid of it. Similarly the $\beta$ - decay gives the nucleus as a whole a mechanism to convert a problematic neutron into a proton, and thereby move closer to a stable nuclide. 


\subsection{Alpha Decay}

Alpha decay involves a cluster of two protons and two neutrons (i.e. helium nucleus) being ejected from a larger nucleus. It does not involve neutrinos, and it is easy to see why: it does not involve any internal reassembly of the protons or neutrons. The reasons for alpha decay are apparent in the Cordus nuclear theory (Pons et al., 2013b), where it is shown that the nucleus comprises networks of nucleons, hence a nuclear polymer. The basic stable unit of this polymer are proton-neutron units, and these fill the edges of connected cubes hence also 2(pn) units. In some of the lighter nuclides there is value in ejecting a $2(\mathrm{pn})$ unit since this permits a more stable configuration for the nuclear polymer, e.g. unstable ${ }_{4} \mathrm{Be}_{4}$ to stable ${ }_{2} \mathrm{He}_{2}$. In the heavy nuclides, alpha decay permits an uncontrollably large nuclear polymer to trim its size towards a more stable configuration. Hence the theory provides a natural explanation for emission of alpha particules. It is primarily a decay caused by instability of the bonds within the nucleus.

\section{Discussion}

\subsection{What Has Been Achieved?}

This work makes a number of novel contributions. The first is ontological, by showing that the NLHV sector can deliver a coherent theory for nuclear decay, with wide-ranging implications. This opens up the field to a very different way of thinking about the decay process, and a reconceptualisation of nucleons and neutrino species. A second contribution is methodological, in the provision of a specific nuclear mechanics, in the form of the HED mechanics, whereby the internal processes of particule interactions may be analysed. This is complementary to Feynman diagrams, but goes deeper. A third contribution is more speculative, but is nonetheless specific and interesting. This is the determination of a plausible NLHV design for the neutrino and antineutrino.

\subsection{Interpretation}

There are a number of puzzling features about the neutrino species, as identified at the outset. We are now at a position to offer explanations for these phenomena, using this new theory.

\subsubsection{Neutrino not Its Own Antiparticle}

The Cordus HED mechanics suggests the neutrino is not its own antiparticle. The reason is that it cannot be converted to an antineutrino solely by the addition of discrete force complexes [ $\uparrow \downarrow]$. Thus the neutrino is predicted not to be a Majorana fermion. By implication neutrinoless double-beta decay will not occur by annihilation. The idea behind neutrinoless double-beta decay is that two neutrons decay simultaneously, producing two antineutrinos. If one antineutrino was able to spontaneously convert into a neutrino, then perhaps the two might annihilate, hence neutrinoless decay. This is currently an area of active research for physics, partly because it may allow the mass of the neutrino to be determined. The present theory forbids the mutual annihilation pathway, though this does not preclude other ways of disposing of the antineutrinos.

\subsubsection{Neutrino Speed}

We can anticipate why the neutrino travels at the speed of light. A neutrino lacks discrete forces in all three emission directions (HEDs), and therefore does not meet reasonable stability criteria. Consequently it borrows discrete forces from the fabric, and is therefore required to move in the fabric. This is the same basic principle for how the photon moves (Pons, 2011). We suggest that the neutrino fills its axis by interacting with the discrete forces of the fabric, thereby obtaining a dynamic stability. The density of fabric discrete forces determines the availability of the fabric to engage in such actions, and hence the fabric density is proposed to determine the speed of light (D. J. Pons \& A. D. Pons, 2013). This also implies that the speed of the neutrino will be dependent on the density of the fabric. In particular, it should slow down in locations where gravitation is stronger or matter is denser. Hence the neutrino appears to show refraction-like behaviour in matter. The Mikheyev-Smirnov-Wolfenstein effect, whereby the oscillation of neutrinos between generations is different in matter and the vacuum (Wolfenstein, 1978), may have a related causality.

\subsubsection{Spin-Hand}

The neutrino is only left-spin-handed. This is strange, because it is the only fermion with this property. All neutrinos are left-spin-handed, and all antineutrinos are right-spin-handed, or at least that is what empirical results suggest. In a QM context left-hand means that the spin of the particle (by the right-hand grip rule) is in the opposite direction to the motion. We use the term left-spin-hand to show that the concept is related to spin, not the matter-antimatter species hand (Pons et al., 2014b).

We interpret the neutrino spin as angular momentum. This suggests that the neutrino always and only has angular momentum in one direction, and the antineutrino in the other. With the Cordus model for these particules, see 
previous figures, we can start to see why the imbalanced interaction with the fabric may result in a preferential movement of the reactive ends, one intimately determined by the sequence of energisation of discrete forces. Thus it is easy to make a conceptual case for causality from the matter-antimatter species differentiation by hand (energisation sequence), also involving the incomplete HED activation of the neutrino species (which also explains their linear motion), through to a rotational reactive moment. Therefore this theory predicts that neutrinos are all left-spin-handed, and that there are no right-spin-handed neutrinos or left-spin-handed antineutrinos. The Cordus theory predicts that we would see a similar spin effect in other particules with imbalanced discrete forces. The only other particule that moves at $\mathrm{c}$ is the photon, and it does not have any hand and therefore the spin effect does not arise there at all.

\subsubsection{Neutrino Mass}

The Cordus explanation for gravitation is that the sequential energisation of the HEDs creates a torsional pulse that is transmitted outwards, and this creates gravitational attraction. Activation of the three HEDs seems necessary for an enduring mass or gravitational effect. The neutrino does not have the necessary complete HEDs to offer its own gravitational interaction: a similar situation to the photon. Therefore this theory predicts that the neutrino has no nominal mass, based on its lack of the necessary structures. However, "mass" may not be quite the right way to look at this. In particular, both the photon and neutrino make up for their incompletely energised HEDs by moving in the fabric. Thus they temporarily do have full HEDs, albeit only instantaneously. Therefore it is possible that they also do have an instantaneous mass and gravitation. While it may register as mass, it would however not be an enduring mass. We conceptualise it rather as an artefact of the propagation process. So it is possible to conceive of the neutrino having zero nominal mass, though a small dynamic mass. This is comparable to the MSW effect (Wolfenstein, 1978) which models the situation as the neutrino obtaining an "effective mass" by a forward scattering process when propagating through matter.

\subsubsection{Trajectory-Bending}

The Cordus theory envisages gravitational bending of the neutrino locus occurring without the particule needing to have mass of any kind. The gravitational bending can instead be explained as the gradient in the fabric density near a large mass, the same explanation as previously given for gravitational bending of light (Pons, 2011). The fabric is slightly denser on the side of the particule nearest the mass, so a discrete force on that side is impeded in its re-energisation, hence the relevant reactive end accomplishes a slightly lesser forward displacement than that closer to the far side, thus bending the trajectory towards the mass. This theory readily accommodates the slowing of neutrinos in denser material, i.e. refraction. This is consistent with the empirical evidence whereby neutrinos are thought to exhibit refraction-like behaviour in their passage through matter.

\subsubsection{Why Are Neutrinos so Unreactive?}

The explanation for why neutrinos react little with matter is that their frequency is too low, and their motion too fast. Reactivity between particules requires that their reactive ends be in the same place and phase at a moment in time, so that the discrete forces have an opportunity to interact. The fast motion of the neutrino, and the presumed relatively large span between of its reactive ends (span is inversely related to frequency or mass in this theory) makes co-location difficult. In a similar way long-wavelength radio waves have greater penetration (less engagement with matter) than visible light.

\subsubsection{Differentiation Between Neutrino and Neutron}

The neutrino and neutron have similar overt discrete force structures, but the neutron has a large additional covert component. These covert discrete forces are balanced, hence obviating the need for the neutron to move like the neutrino, and numerous, hence increasing its mass. (Note that the allocation of discrete forces is merely nominal in the present theory.) The neutrino is, by comparison, a minimalist particule: it has the NLHV structure and a functional energisation sequence, hence a matter-antimatter species differentiation (which the photon lacks), but only a few discrete forces and hence not much energy. From the Cordus perspective, a fundamental particule is one that overtly displays all its discrete forces. Examples would then be the photon, electron, and electron neutrino. All other stable particules are predicted to have covert components, including the quarks, proton, and neutron.

\subsubsection{What Purpose do Neutrinos Serve?}

The primary answer appears to be: They remove waste matter-antimatter hand structures from assemblies of particules, thereby helping the remanufacturing process. They may also actively contribute to the decay of the nuclides. 


\subsection{Limitations of This Theory}

This work is conjectural in nature, and the resulting theory is conceptual. This is because it is a gedanken experiment. The theory is not yet at the point of having a mathematical formalism, and this limits the ability to make quantitative predictions or check the mathematical integrity of the ideas. We acknowledge those as necessary future endeavours.

\subsection{Implications for Further Research}

Having a theory for the inner structure of neutrinos permits further exploration of the many neutrino phenomena. For example, there are significant implications for neutrino involvement in asymmetrical baryogenesis.

\section{Conclusions}

A novel conceptual theory has been developed for the neutron decay processes, based on a NLHV design. A core concept is that the type of particule (proton, neutron, etc.) has a characteristic emission of discrete forces, and that changes to the emissions cause the particule to be changed to another type. We refer to this as a remanufacturing process, which is an especially relevant term given that the processes may involve change to the hand structure that defines the matter-antimatter species. This new mechanics is able to correctly predict the outcomes of the various decay processes, beta minus, beta plus, and electron capture. It also predicts NLHV structures for the neutrino and antineutrino. These structures naturally give rise to explanations for the velocity of these particules, and their selective spin.

The overall outcome is a new conceptual foundation for the decay processes, one based in a NLHV design. It is an ontologically rather than mathematical solution, and while it lacks a quantitative formalism, it compensates in explanatory power. It offers a logically consistent set of explanations for a wide variety of decay phenomena. The larger Cordus theory, of which this is a coherent part, extends the explanations to a yet wider range of phenomena in fundamental physics, and demonstrates the viability and vitality of NLHV solutions.

\section{Contribution Statement}

All authors contributed to the development of the concepts, DP devised the HED mechanics and the neutrino models.

\section{References}

Bell, J. S. (1964). On the Einstein Podolsky Rosen Paradox. Physics, 1(3), 195-200. Retrieved from http://philoscience.unibe.ch/documents/TexteHS10/bell1964epr.pdf

Bohm, D., \& Bub, J. (1966). A proposed solution of measurement problem in quantum mechanics by a hidden variable theory. Reviews of Modern Physics, 38(3), 453-469. http://dx.doi.org/10.1103/RevModPhys.38.453

Chadwick, J. (1932). Possible Existence of a Neutron. Nature, 129(3252), 312. http://dx.doi.org/10.1038/129312a0

de Broglie, L. (1925). Recherches sur la théorie des quanta (Researches on the quantum theory). Annales de Physique, 3(10). Retrieved from http://tel.archives-ouvertes.fr/docs/00/04/70/78/PDF/tel-00006807.pdf

De Zela, F. (2008). A non-local hidden-variable model that violates Leggett-type inequalities. Journal of Physics A: Mathematical and Theoretical, 41(50), 505301. http://dx.doi.org/10.1088/1751-8113/41/50/505301

Fermi, E. (1934). Versuch einer Theorie der $\beta$-Strahlen. Zeitschrift für Physik, 88(3-4), 161-177. http://dx.doi.org/10.1007/BF01351864

Gisin, N. (2011). Impossibility of covariant deterministic nonlocal hidden-variable extensions of quantum theory. Physical Review A, 83(2), 020102. http://dx.doi.org/10.1103/PhysRevA.83.020102

Groblacher, S., Paterek, T., Kaltenbaek, R., Brukner, C., Zukowski, M., Aspelmeyer, M., \& Zeilinger, A. (2007). An experimental test of non-local realism. Nature, 446(7138), 871-875. http://dx.doi.org/10.1038/nature05677

Laudisa, F. (2008). Non-Local Realistic Theories and the Scope of the Bell Theorem. Foundations of Physics, 38(12), 1110-1132. http://dx.doi.org/10.1007/s10701-008-9255-8

Leggett, A. (2003). Nonlocal Hidden-Variable Theories and Quantum Mechanics: An Incompatibility Theorem. Foundations of Physics, 33(10), 1469-1493. http://dx.doi.org/10.1023/a:1026096313729

Murayama, H. (2002). Origin of Neutrino mass. Physics World, May, 35-39. Retrieved from http://physicsworldarchive.iop.org/full/pwa-pdf/15/5/phwv15i5a36.pdf 
Pauli, W. (1930). Letter to the Physical Institute of the Federal Institute of Technology.

Pons, D. J. (2011). Contrasting internal structures: Photon and electron. Vixra, 1109.0045, 1-9. Retrieved from http://vixra.org/abs/1109.0045

Pons, D. J., \& Pons, A., D. (2013). Outer boundary of the expanding cosmos: Discrete fields and implications for the holographic principle. The Open Astronomy Journal, 6, 77-89. http://dx.doi.org/10.2174/1874381101306010077

Pons, D. J., Pons, A. D., \& Pons, A. J. (2013a). Time: An emergent property of matter. Applied Physics Research, 5(6), 23-47. http://dx.doi.org/10.5539/apr.v5n6p23

Pons, D. J., Pons, A. D., \& Pons, A. J. (2013b). Explanation of the Table of Nuclides: Qualitative nuclear mechanics from a NLHV design. Applied Physics Research, 5(6), 145-174. http://dx.doi.org/10.5539/apr.v5n6p145

Pons, D. J., Pons, A. D., \& Pons, A. J. (2013c). Synchronous interlocking of discrete forces: Strong force reconceptualised in a NLHV solution. Applied Physics Research, 5(5), 107-126. http://dx.doi.org/10.5539/apr.v5n5p107

Pons, D. J., Pons, A. D., \& Pons, A. J. (2014a). Annihilation mechanisms. Applied Physics Research, 6(2), 28-46. http://dx.doi.org/10.5539/apr.v6n2p28

Pons, D. J., Pons, A. D., \& Pons, A. J. (2014b). Differentiation of Matter and Antimatter by Hand: Internal and External Structures of the Electron and Antielectron. Physics Essays, 27, 26-35. http://vixra.org/abs/1305.0157

Pons, D. J., Pons, A. D., Pons, A. M., \& Pons, A. J. (2012). Wave-particle duality: A conceptual solution from the $\begin{array}{lllll}\text { cordus } \quad \text { conjecture. } & \text { Physics }\end{array}$ http://physicsessays.org/doi/abs/10.4006/0836-1398-25.1.132

Romero, J., Leach, J., Jack, B., Barnett, S. M., Padgett, M. J., \& Franke-Arnold, S. (2010). Violation of Leggett inequalities in orbital angular momentum subspaces. New Journal of Physics, 12(12), 123007. http://dx.doi.org/10.1088/1367-2630/12/12/123007

Rutherford, E. (1911). The Scattering of $\alpha$ and $\beta$ Particles by Matter and the Structure of the Atom. Philosophical Magazine, 6(21), 669-688. http://dx.doi.org/10.1080/14786435.2011.617037

Thomson, J. J. (1904). On the Structure of the Atom: an Investigation of the Stability and Periods of Oscillation of a number of Corpuscles arranged at equal intervals around the Circumference of a Circle; with Application of the Results to the Theory of Atomic Structure. Philosophical Magazine, series 6, 7(39), 237-265. http://dx.doi.org/10.1080/14786440409463107

Wilson, F. L. (1968). Fermi's theory of beta decay. American Journal of Physics, 36(12), 1150-1160. http://dx.doi.org/10.1119/1.1974382

Wolfenstein, L. (1978). Neutrino oscillations in matter. Physical Review D, 17(9), 2369-2374. http://dx.doi.org/10.1103/PhysRevD.17.2369

\section{Copyrights}

Copyright for this article is retained by the author(s), with first publication rights granted to the journal.

This is an open-access article distributed under the terms and conditions of the Creative Commons Attribution license (http://creativecommons.org/licenses/by/3.0/). 